\title{
Hydrogen to the rescue
}

\author{
Large-scale, environmentally friendly hydrogen production will rely on steam methane reforming coupled with \\ carbon capture and electrolysis, but solar fuels could have a disruptive role to play.
}

$\mathrm{H}$ ydrogen is considered an environmentally friendly replacement for fossil fuels as reaction with oxygen only produces $\mathrm{H}_{2} \mathrm{O}$. However, although hydrogen is ubiquitous, on Earth it is bound up in compound form, such as $\mathrm{H}_{2} \mathrm{O}$ or fossil fuels. Forming molecular hydrogen conventionally requires large amount of energy, which mitigates the environmental benefits and is not sustainable in the long term ${ }^{1}$.

Recently, a Royal Society policy report reviewed the possibility of a hydrogenbased economy by analysing the relative advantages of different hydrogen production approaches against practical barriers associated with scalability, storage and distribution ${ }^{2}$. In order of technological readiness, the report focusses on steam methane reforming, electrolysis, biological methods and solar to fuel approaches. Smallscale storage, which requires solving its own set of challenges, is not discussed in depth.

Currently, the majority of hydrogen produced worldwide is devoted to production of ammonia for fertilizer synthesis. As hydrogen production was 60 million tonnes in $2017^{3}$, much less than oil production, this does not meet worldwide energy production. The vast majority is generated by steam methane reforming, whereby methane reacts with water over oxide-supported transition metal catalysts to produce syngas, a mix of $\mathrm{CO}$ and $\mathrm{H}_{2}$. This requires high temperatures generated using fossil fuels that creates $\mathrm{CO}_{2}$, needing carbon capture and storage to reduce environmental impact. Industrial catalyst choice is determined by a mix of cost and activity. Materials science can help by enabling a more complete understanding of catalyst reaction mechanisms, enabling choice of selective, cheap and stable materials. Moreover, syngas separation is energy intensive and conventionally achieved either by pressure swing or cryogenic swing adsorption. Membrane approaches for separations, such as preferential adsorption in porous materials or molecular sieving, may also be applied by judicious choice of materials.

A cleaner approach, and one used commercially, is to electrochemically generate hydrogen by water splitting (pictured), evolving hydrogen and oxygen ${ }^{4}$. Alkaline electrolysers are most mature, although polymer-based acidic electrolysers that offer higher currents and better load following capability are increasingly emerging. Solid oxide electrolysers that operate at high temperatures potentially have better efficiencies, but a carbonneutral heat source is needed, and hightemperature operation has its own set of challenges.

Electrolysers need significant energy to evolve hydrogen as kinetics are slow at ambient temperature. Electrocatalysts can increase kinetics. Ideally, renewable energy would be used to power these reactions. This can be accomplished either by using solar or wind to power electrolysers, or by directly harvesting light in a photoelectrochemical solar fuels approach ${ }^{5}$.

Research on electrocatalysts focusses on optimizing materials that promise similar performance, in terms of metrics such as exchange current densities, to benchmark (but expensive) platinum. There is much activity on carbon-supported and iron-based electrocatalysts ${ }^{6}$, as well as engineering layered materials to expose active sites ${ }^{7}$.

Photoelectrocatalyst devices are in principal simpler than electrocatalyst devices, with charge separation induced directly by the solid-liquid interface. However, this comparative simplicity provides less freedom for optimization, as electrocatalyst devices currently have much higher overall efficiencies. Indeed, photoelectrocatalyst optimization is a challenging problem, involving appropriate material combinations, often based on metal cocatalysts supported on metal oxides, which enable good electron transport without enabling side reactions ${ }^{8}$, and with careful control of morphology to optimize light harvesting and photoelectrochemical performance?

Even if hydrogen is cleanly produced, this is only one of several barriers that must be surmounted; other infrastructure requirements must be solved, such as transport, possibly by refitting gas pipelines, and associated large-scale temporary storage, potentially in salt caverns. This may be feasible in one place, but as infrastructure and geological structures are unevenly distributed, hydrogen uptake will be dependent on local resources.

The report concludes that electrolysis or photoelectrolysis approaches that split

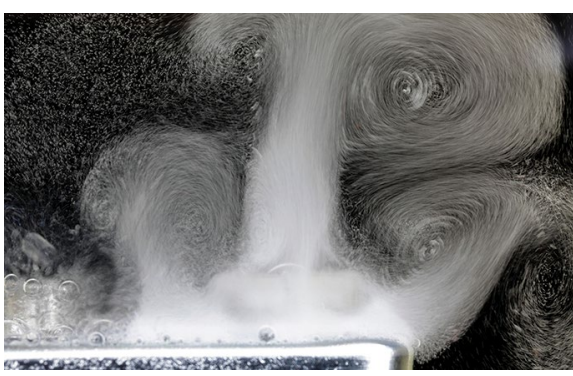

Credit: Durk Gardenier/Alamy Stock Photo

water would be a cleaner alternative to steam methane reforming, with electrolysis the more mature approach, possibly supplemented by biological production ${ }^{2}$. To generate significant amounts of hydrogen by electrolysis or photoelectrolysis, it is necessary not only to develop cheap, stable and efficient materials but also to develop mechanistic understanding. It remains to be seen whether the more innovative electrocatalysts can be implemented in devices at industrial scales sufficient to meet energy demands, let alone photoelectrocatalytic devices that by comparison are still in their infancy.

Overall, there are significant challenges to a hydrogen-based economy, and as a switch away from fossil fuels it will be part of a mix of options, one of a range of replacement energy vectors alongside other fuel-cell and battery technologies. However, it is clear that the search for better hydrogen production technologies has driven a range of fundamental and applied materials research. We cannot say where this will ultimately lead, but we look forward to covering future developments.

Published online: 25 June 2018 https://doi.org/10.1038/s41563-018-0129-y

References

1. Chu, S., Cui, Y. \& Liu, N. Nat. Mater. 16, 16-22 (2017).

2. Options for Producing Low-Carbon Hydrogen at Scale (Royal Society, 2018); https://go.nature.com/2ylnHSZ

3. Philibert, C. Renewable Energy for Industry (IEA, 2017); https://go.nature.com/2LFwbkJ

4. Stamenkovic, V. R., Strmcnik, D., Lopes, P. P. \& Markovic, N. M. Nat. Mater. 16, 57-69 (2017).

5. Montoya, J. H. et al. Nat. Mater. 16, 70-81 (2017).

6. Lefèvre, M., Proietti, E., Jaouen, F. \& Dodelet, J. P. Science 324, 71-74 (2009).

7. Li, H. et al. Nat. Mater. 15, 48-53 (2016).

8. Wang, Q. et al. Nat. Mater. 15, 611-615 (2016).

9. Kibria, M. G. et al. Nat. Commum. 6, 6797 (2015). 